\author{
Thomas Daiber \\ (iD https://orcid.org/0000-0003-4185-294X \\ Institut für Slavistik \\ Justus-Liebig-Universität \\ D - 35394 Gießen, Otto-Behaghel-Straße 10 D \\ thomas.daiber@slavistik.uni-giessen.de \\ https://doi.org/10.18778/8220-520-6.02
}

\title{
ALTKIRCHENSLAVISCH „HAKO И“ ALS MITTEL DES HYPOTHETISCHEN VERGLEICHS
}

\author{
Old Church Slavonic "iako h" as marker \\ of a hypothetical comparison \\ Аревнецерковнославянское сочетание «tАко h» \\ как среАство гипотетического сравнения
}

\begin{abstract}
Summary
The paper discusses the semantics of the Old Church Slavonic syntactic compound "rкко u", which appears in translations regularly as equivalent of a Greek compound "com-

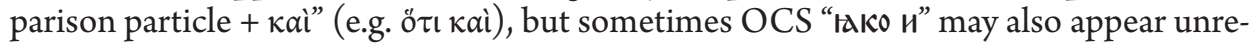
lated to an underlying Greek pattern. On the material of OCS Codex Marianus the paper documents all instances of "IAко $и$ " together with its Gr equivalents (2), compares the findings with similar use of "'ako $\mathbf{u}$ " in other Church Slavonic texts (3) and formulates the hypothesis (4), that starting from OCS "זیко и" took on the meaning of a hypothetical comparison ("as if").
\end{abstract}

\section{Резюме}

В статье рассматривается вопрос о значении Аревнецерковнославянского сочетания «اАко и», которое появмяется, как правило, в перевоАной митературе эквивалентно с сочетаниями «сравнительная частица + каџ» в греческом подлиннике

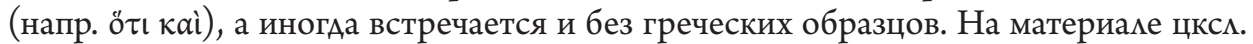
Мариинского Евангелия в статье воспроизводятся текстовые фрагменты с сочетанием «اءсо и» вместе с их графическими формами (2), на основании которых указывается несколько похожих фрагментов в Аругих цксл. текстах (3) и ставится гипотеза (4), согласно которой, исходя из цксл. употребления сочетания «ґАко и», оно приобретало значение гипотетического сравнения («как будто»). 


\section{Streszczenie}

Artykuł rozpatruje kwestię znaczenia staro-cerkiewno-słowiańskiego określenia „ґঝко и” występującego z reguły w literaturze tłumaczonej na równi z ekwiwalentem „par-

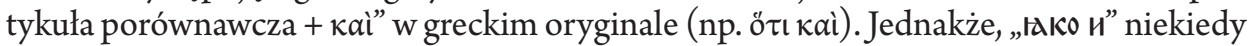
występuje w oderwaniu od kanonicznych wzorców greckich. Na podstawie cerkiewnosłowiańskiego Kodeksu Mariańskiego w artykule zostały uwzględnione wszystkie fragmenty z określeniem „ıксо и” łącznie z formami greckimi (2), na podstawie których zostały poświadczone podobne fragmenty w innych tekstach greckich (3), a także została postawiona hipoteza (4), w świetle której użycie określenia „ґঝко и” czasami przybierało kontekst porównawczy („как буАто”).

Ключевые слова: церковнославянский, ғако и, гипотетическое сравнение.

Keywords: Church Slavonic, tako $\mathrm{n}$, hypothetical comparison.

Słowa kluczowe: cerkiewnosłowiański, tako n, hipotetyczne porównanie.

\section{Fragestellung}

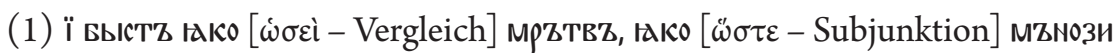

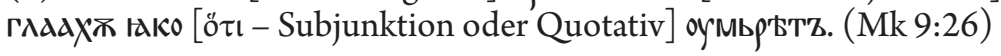

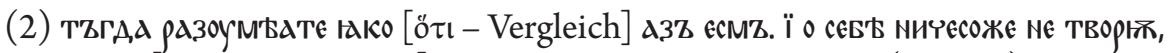

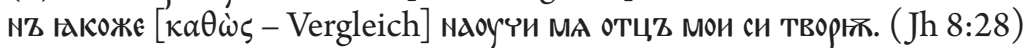

Die einleitenden Zitate $(1+2)$ aus dem Codex Marianus ${ }^{1}$ zeigen, dass altkir-

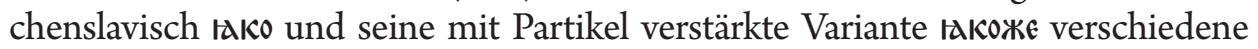
griechische Vergleichskonjunktion wiedergeben und auch deren Funktion als Subjunktion oder gegebenenfalls (das Zitat 1 ist hierfür aber nicht beweiskräftig) als Markierung der Redewiedergabe (hoti bzw. jako recitativum; Daiber, 2009, 2013) übernehmen können. In diesem Beitrag soll es um die im Aksl. öfters auftretende Verbindung ז ғкс $и=$, wie und/auch“ gehen ${ }^{2}$. Dabei wird zu fragen sein, ob die Ver-

1 Die ursprünglich glagolitische Handschrift wurde in dem hier benutzten CCMH (1986-2010) nach Jagič (1960) digitalisiert und schreibt ько; aus Gründen der Vereinheitlichung normalisiere ich zu rako. Supralineare Buchstaben werden in diesem Beitrag kommentarlos auf die Linie gestellt, aber Abkürzungen werden nicht aufgelöst. Abkürzungszeichen, gr. Behauchungs- und Akzentzeichen sind entfernt, die Interpunktion ist ggf. normalisiert worden.

2 An Literatur speziell zu rako (и) ist mir nur Rondestvedt (1986, 148f.) bekannt, rako im Rahmen der Determinationsproblematik erwähnt, aber den Unterschied zu rako и nicht thematisiert. - Anstatt von einer недостоверное сравнение (Tommola, 2014) benutzen wir in diesem Aufsatz mit Romanova (2008, 17 et passim) lieber den Terminus гипотетические сравнение. 
bindung raке $\mathrm{n}$ als individuell motivierte oder von der gr. Übersetzungsvorlage ausgelöste Übersetzungsvariante von alleinigem rako zu werten ist, oder ob rako $\mathrm{n}$ tendenziell im Aksl. dazu neigt, einen hypothetischen Vergleich auszudrücken. Zur Beantwortung dieser Frage betrachten wir (Abschnitt 2) die Entsprechungen von IAKo и im aksl. Codex Marianus (einem fast vollständigen Tetraevangelium), vergleichen den Befund mit Stellen aus anderen kirchenslavischen Texten (Abschnitt 3) und bilden eine vorsichtige Hypothese (Abschnitt 4).

\section{Die Verbindung taко и im Codex Marianus}

Bei einem Übersetzungsvergleich, der darauf abzielt, Abweichungen der Übersetzung vom Original auf ihre mögliche semantische oder grammatische Funktion zu untersuchen, wird man gut daran tun, textologisch unsichere Stellen auszusondern, die gesicherten regelmäßigen Übersetzungsäquivalente als das Unmarkierte von den Abweichungen abzusondern und dann die semantische Tendenz der Abweichungen (denn regelmäßige Abweichungen ${ }^{3}$ kann es nicht geben) anzudeuten. Der methodische Versuch ist dahingehend beschränkt, dass er sich im Wesentlichen auf nur eine aksl. Übersetzung des Bibeltextes stützt, jedoch (in Fußnoten) wenigstens orientierend auf die Übersetzungsvarianz in anderen aksl. Bibeltexten aufmerksam macht. Die sich stellende Frage, ob rako $\mathbf{n}$ eine Bedeutungsveränderung durchläuft, welche in den aksl. Verhältnissen bereits angelegt ist, kann im Rahmen eines Aufsatzes nicht beweiskräftig beantwortet, die Frage an sich aber plausibel gemacht werden.

\subsection{Das Material}

Die längere Tabelle (2.1c) enthält in der linken Spalte die von Cejtlin, Večerka, Bláhová (1994, 793-797) für aksl. ı «ko angegebenen gr. Äquivalente, die im gr. Evangelientext (digitalisiert in SBL, 2010) aufgesucht wurden. Die rechte Spalte enthält mit Angabe der Fundstellen die jeweiligen aksl. Äquivalente, wie sie im Marianus (digitalisiert in CCMH, 1986-2010) erscheinen. Bei den gr.

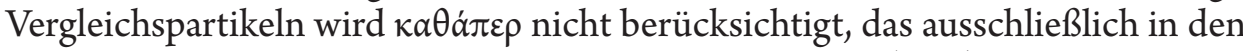
Episteln vorkommt, und natürlich verzichtet die Tabelle (2.1c) auf die den Platz

${ }^{3}$ Die regelmäßige Abweichung von der Regel ist selbst eine Regel, und sollte sich eine Übersetzung auch durch höchst extreme, aber regelmäßig auftretende Übersetzungslösungen von allen anderen Übersetzungen ihrer Zeit unterscheiden, so ist sie zwar eine Abweichung von der allgemeinen Norm, aber textimmanent eben doch geregelt. Uns interessieren hier die Abweichungen, die weder im Vergleich zu einer allgemeinen Norm noch textimmanent geregelt genannt werden können, aber deshalb dennoch nicht zufällig sein müssen. 
sprengenden Nachweise von einfachem $\omega \varsigma^{4}$, ó $\tau \iota$ und $\pi \tilde{\omega} \varsigma^{5}$. Die Entsprechungen von ıако и іm Marianus mit $\dot{\omega} \zeta$ ŏ $\tau$ (2.1a) bzw. den mit $\dot{\omega} \zeta$ in den gr. Hss. variierenden $\omega \sigma \pi \varepsilon \rho$ und $\omega \sigma \varepsilon \grave{~(2.1 b) ~ w u r d e n ~ i m ~ G e g e n s a t z ~ z u ~ d e n ~ a n d e r e n ~ F u n d s t e l l e n ~}$ (2.1c) nicht über den gr. Text gesucht, sondern vielmehr über die direkte Suche von ґако и im aksl. Text. Natürlich hätte nur eine einzige Tabelle im aksl.> gr. Vergleich (wie $2.1 \mathrm{a}+2.1 \mathrm{~b}$ ) angefertigt werden können, in welcher ausschließlich die gr. Äquivalente für ґґко и gestanden hätten. Mir schien aber sinnvoll, auch einen gr. > aksl. Vergleich (wie 2.1c) dazu zugeben, um die typische Semantik der gr. Vergleichskonjunktionen zu dokumentieren.

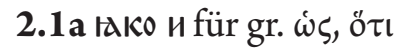

\begin{tabular}{|c|c|}
\hline$\dot{\omega}$ & 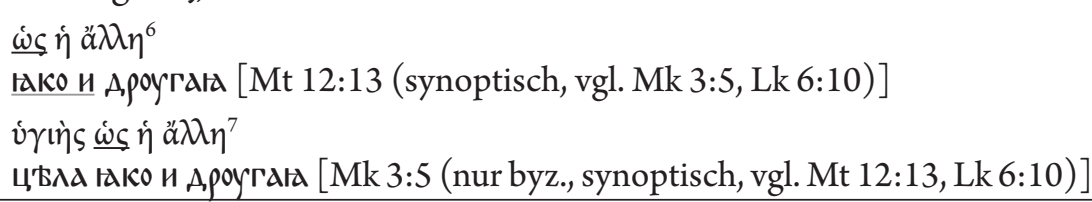 \\
\hline ö $\tau ا$ & 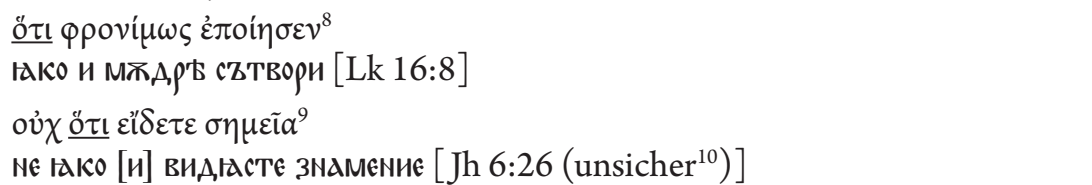 \\
\hline
\end{tabular}

${ }^{4}$ Dies bedingt, dass die Tabelle 2.1c die Vorkommen von bspw. $\omega \sigma \varepsilon \grave{~ n u r ~ n a c h ~ d e r ~}$ Ausgabe SBL angibt, wo durch etwa Mk 1:10 АХХZ ґако ГОлжБь сZХОААШТЬ NA NЬ übergangen wird, wo je nach gr. Hs $\dot{\omega} \zeta$ oder $\dot{\omega} \sigma \varepsilon i$ steht (SBL: $\dot{\omega} \zeta)$. Der Aufsatz beschäftigt sich aber nicht mit dem Vorkommen der gr. Vergleichskonjunktionen und ihrer Varianten, sondern mit aksl. rako (и), und für diesen Zweck ist es ausreichend, die gr. Partikeln und ihre Übersetzungsäquivalente anhand einer gr. Redaktion (SBL) aufzusuchen, während bei der umgekehrten Suche (welche gr. Partikeln entsprechen rako и) dann auch das Schwanken der gr. Hss. im Partikelgebrauch erkannt wird.

5 Aksl. rako (и) erscheint im Codex Marianus nie für gr. $\pi \tilde{\omega} \varsigma$, vielmehr steht für $\pi \tilde{\omega} \varsigma$ immer како (Ausnahmen: Mk 4:40 folgt der byz. Lesart $\tau i$ vı statt $\pi \tilde{\omega} \zeta$; in Lk 12:56 ist $\pi \tilde{\omega} \varsigma$ ausgelassen). П $\tilde{\omega}$ tritt auf bei verbalen (etwa Mk 11:18, Lk 12:11) Vergleichen, hat also adverbialen Charakter (,auf welche Weise, wie“) und bildet als $(\ddot{\eta}) \pi \tilde{\omega} \varsigma=(\Lambda и)$ како die übliche Einleitung einer Frage (etwa Mt 12:29, 21:20).

6 So (ohne kai) der nicht in allen gr. Hss vorfindliche Vergleich in Nestle, Alland (2012, 33), keine Lesarten in Robinson, Pierpont (2005).

7 Der gr. (zusätzliche) Halbvers findet sich nicht in Nestle, Alland (2012), ist aber (als Lesart ohne weitere Varianten) in Robinson, Pierpont $(2005,74)$ dokumentiert.

${ }^{8}$ Keine Lesarten in Nestle, Alland (2012) und Robinson, Pierpont (2005).

9 Ibidem.

10 Jagič $(1960,338)$ liest das и als Ersatz von ursprünglichem А, („В подлиннике бымо да выскоблено“). 


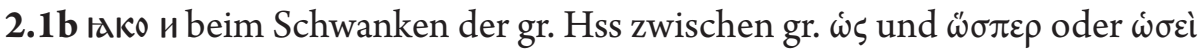

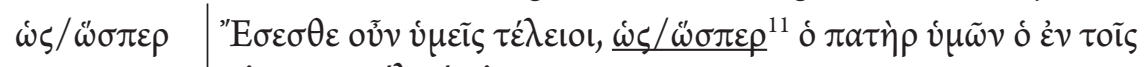

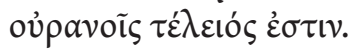

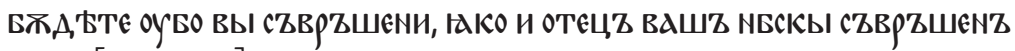
ecTZ. [Mt 5:48]

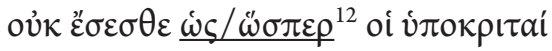

ї ЕГАА МОАИШИ СА NЕ БЖА, ґАКО и АИЦем'Бри [Mt 6:5]

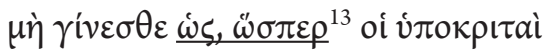

NE Бж, ВТЕ ґако и иПокрити [Mt 6:16]

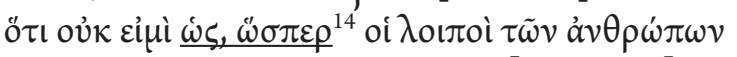

זАКО N'СCMZ זАКо и прочии чАВци [Lk 18:11]

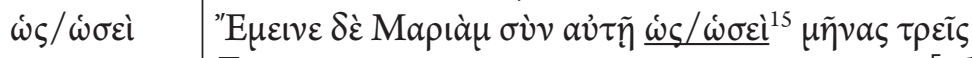

Пр'ББыстZ Же марига сZ нењ Іако и три мсцА [Lk 1:56]

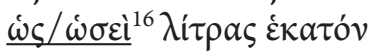

Іако и китрz сZто [Jh 19:39]

2.1c Gr. Vergleichskonjunktionen und ihre aksl. Entsprechung

$\omega ̋ \varsigma \kappa \alpha \grave{~ „, w i e ~}$ auch“"

rakк и (4) [Mt 6:12, 18:33 ( 6:10 ${ }^{18}$ (synoptisch, vgl. Mt 12:13, Mk 3:5)] гакоже и (1) [Lk 9:54 ${ }^{19}$ ]

11 Nestle, Alland $(2012,13)$ und Robinson, Pierpont $(2005,10)$ haben nur die Variante $\dot{\omega} \zeta / \ddot{\omega} \sigma \pi \varepsilon \rho$ (ohne $\kappa \alpha i)$.

12 Nestle, Alland $(2012,14)$ ohne Angabe des Wechsels der Vergleichskonjunk-

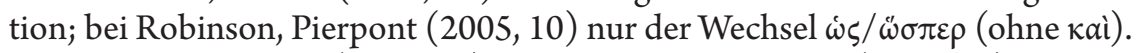

13 Nestle, Alland $(2012,15)$ und Robinson, Pierpont $(2005,11)$ verzeichnen nur den Wechsel $\dot{\omega} \varsigma / \omega \sigma \pi \varepsilon \rho$ (ohne $\kappa \alpha \grave{)}$.

14 Nur Nestle, Alland $(2012,259)$ kennen die eher seltene Variante $\omega \varsigma$ für $\omega \sigma \pi \varepsilon \rho$.

15 Nestle, Alland $(2012,181)$ und Robinson, Pierpont $(2005,120)$ vermerken nur den Wechsel $\dot{\omega} \zeta / \hat{\omega} \sigma \varepsilon \grave{~(o h n e ~ \kappa a i ̀) . ~}$

16 Nestle, Alland (2012) ohne Lesart, Robinson, Pierpont (2005, 243) vermerken

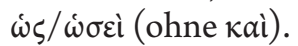

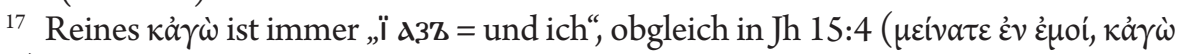
¿่v v่ũv) der Imperativ „bleibet“" am ehesten den Vergleich „wie ich“ nach sich ziehen sollte.

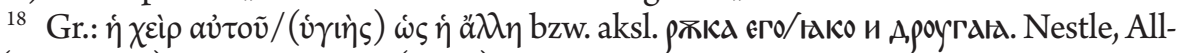
and $(2012,202)$ verzeichnen $\omega \varsigma$ (+ $\kappa \alpha \iota) \eta \alpha \lambda \lambda \eta$ oder $v \gamma \downarrow \eta \varsigma \omega \varsigma \eta \alpha \lambda \lambda \eta$, Robinson, Pierpont (2005, 133) vүı५ $\omega \varsigma \eta \alpha \lambda \eta \eta)$ als mögliche Zusätze, weshalb wegen nicht auszuschließender Beteiligung von кà der aksl. Beleg als Äquivalent für $\ddot{\omega} \varsigma$ kà einzuordnen ist.

19 Nestle, Alland $(2012,223)$ und Robinson, Pierpont $(2005,148)$ verzeichnen nur $\omega \varsigma \varsigma$ kà als Beginn des Zusatzes. 


\begin{tabular}{|c|c|}
\hline $\begin{array}{l}\text { ó } \tau \text { k kà̀ „und } \\
\text { auch“ }\end{array}$ & $\begin{array}{l}\text { Iaко и (5) [Mk 4:41 „sowohl als auch“, Lk 4:43 „quotativ } \\
\text { dass: Auch“, Lk 8:25 „sowohl als auch“, Jh 6:36 „und auch“, Jh } \\
\text { 8:25 „wie auch“] } \\
\text { IAко [Mt 8:27 „sowohl als auch“, Mk 9:13 „und auch“] }\end{array}$ \\
\hline $\begin{array}{l}\text { ö } \pi \omega \varsigma \text { „auf } \\
\text { welche Wiese } \\
\text { (meist final)“ }\end{array}$ & $\begin{array}{l}\text { Ad (Mt 5:45, 6:2, 6:4, 6:5, 6:16, 6:18, 8:17, 8:34, 9:38, 13:35, } \\
\text { 22:15, 23:35, Lk 2:35, 7:3, 10:2, 11:37, Jh 11:57) } \\
\text { КАко (Mt 12:14, Mk 3:6, Lk 24:20) } \\
\text { Іако А, (Mt 26:59, Lk 16:26, 16:28) }\end{array}$ \\
\hline 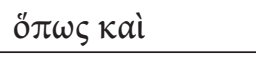 & $\varnothing$ \\
\hline $\begin{array}{l}\text { ö } \sigma \pi \varepsilon \rho \text { „auf wel- } \\
\text { che Weise“ }\end{array}$ & $\begin{array}{l}\text { гако [Mt 6:2, 12:40, 13:40, 18:17, 24:38, Lk 17:24, Jh 5:21] } \\
\text { гакожє }[\text { Mt 6:7, 20:28, 24:27, 24:37, 25:14, 25:32, Jh 5:26] }\end{array}$ \\
\hline$\omega \sigma \pi \varepsilon$ & $\varnothing$ \\
\hline 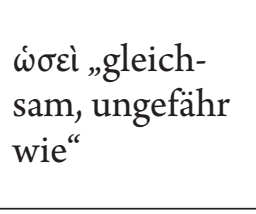 & 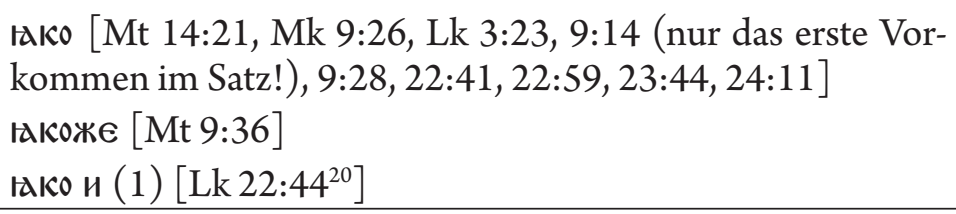 \\
\hline$\dot{\omega} \sigma \varepsilon \grave{~ \kappa \alpha i ̀ ~}$ & $\emptyset$ \\
\hline$\pi \tilde{\omega} \varsigma \kappa \alpha i$ & $\emptyset$ \\
\hline 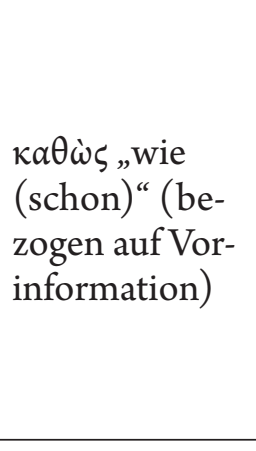 & 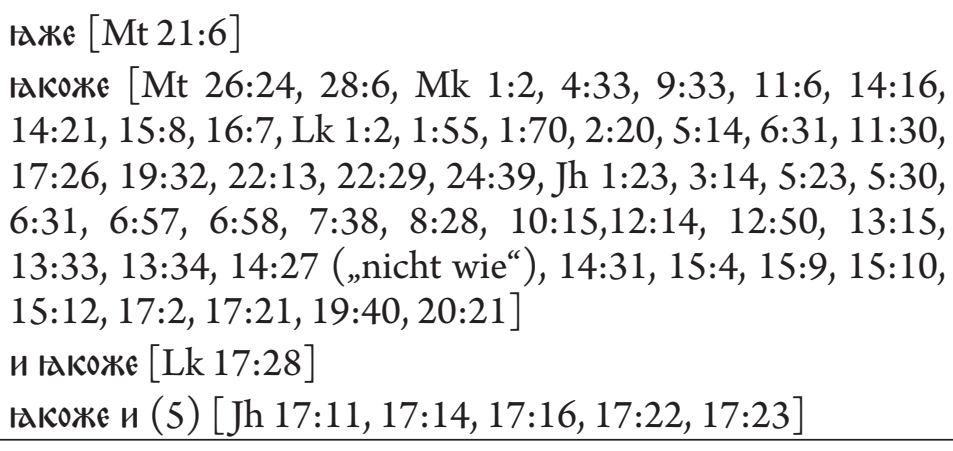 \\
\hline 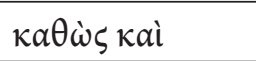 & Іакоже и (3) [Lk 6:36 $\left.{ }^{21}, \mathrm{Lk} 11: 1^{22}, \mathrm{Lk} 24: 24\right]$ \\
\hline
\end{tabular}

${ }^{20}$ Keine Lesarten in Nestle, Alland (2012) und Robinson, Pierpont (2005).

${ }^{21}$ Nestle, Alland $(2012,205)$ und Robinson, Pierpont $(2005,153)$ haben je $\kappa a \theta \omega \varsigma$ (каi) als Lesart.

${ }^{22} \mathrm{Im}$ Codex Marianus steht nur über einen Zeilenumbruch hinweg ғакоже/ ноАNZ, so dass ursprüngliches * ғакожє и ноднz für überall (Nestle, Alland 2012 und Robinson,

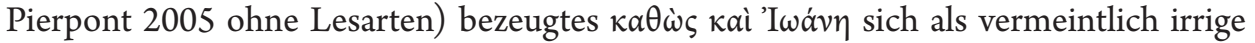
Doppelung leicht verlieren konnte. Wegen methodischer Unsicherheit ist das aksl.

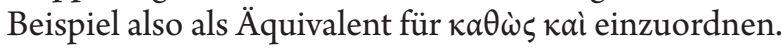




\begin{tabular}{|c|c|}
\hline$\kappa a \theta \grave{a}$ & Іакоже [Mt 27:10] \\
\hline Sió $\tau \iota$, um“ (final) & 3Анe [Lk 1:13, 2:7, 21:28] \\
\hline
\end{tabular}

\subsection{Semantik und Funktion}

Es ist aus den Tabellen (2.1a-c) ersichtlich, dass das Vorkommen von rako $\boldsymbol{и}$ und ґкожє $\boldsymbol{и}$ im aksl. Evangelientext verschieden motiviert sein kann. An manchen Stellen übersetzt ґако и bzw. ґакожє и wörtlich die entsprechende gr. Wendung „Vergleichskonjunktion $+\kappa a i$ “ und ist damit als distinguierendes Material für eine Untersuchung genuin aksl. Ausdrucksweise nicht aussagekräftig. Zur besseren Orientierung wurde in Tabelle 2.1c die Summe der Fundstellen von זАко/ґакожє и је in runden Klammern den Fundorten in der Bibel vorangestellt. So ergibt sich: Das $20^{23} \mathrm{Mal}$ im Codex Marianus auftretende tako $\mathbf{n}$ ist $9 \mathrm{Mal}$ äquivalent mit einer gr. Verbindung „Vergleichspartikel + $\kappa a i$ “, bei ґакожє $и$ ist dies $4 \mathrm{Mal}$ von insgesamt neunmaligem Auftreten der Fall. Die vom gr. Original motivierten Stellen brauchen hier nicht weiter betrachtet werden, sind allerdings nicht ohne Aufschlusswert, denn sie zeigen, dass nicht alle gr. Vergleichskonjunktionen mit kaì kombiniert werden (es fanden sich nicht, ungeachtet semantischer Wahrscheinlichkeit, die Vergleichskonjunktionen ö $\pi \omega \varsigma / \tilde{\omega} \sigma \pi \varepsilon \rho / \dot{\omega} \sigma \varepsilon \dot{l} / \pi \tilde{\omega} \varsigma+\kappa \alpha \dot{i})$, was wichtig ist für die Interpretation jener Stellen $(2.1 \mathrm{~b})$, die offenkundig mit einem Schwanken der gr. Hss zwischen $\dot{\omega} \zeta$ und $\ddot{\omega} \sigma \pi \varepsilon \rho / \dot{\omega} \sigma \varepsilon \dot{~ z u}$ tun haben.

Es bleiben also 11 (ґко и) + 5 (ґккжє и) Bibelstellen, wo der aksl. Wortlaut nicht mit Vorhandensein der gr. Konjunktion kaì erklärt werden kann. Die 11 Stellen, wo tako $\mathrm{n}$ ohne Bezug auf gr. kai auftaucht, zerfallen in zwei Kategorien: auf der einen Seite tritt tako и als Äquivalent einer reinen

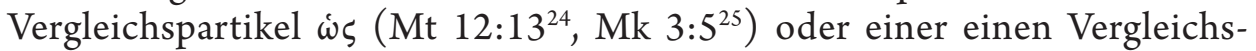
anschluss vorbereitenden Subjunktion (Lk 16:8 ${ }^{26}$, Jh 6:26 $6^{27}$ ) auf, worunter eine Stelle (Jh 6:26) textuell zweifelhaft ist; auf der anderen Seite tritt rako

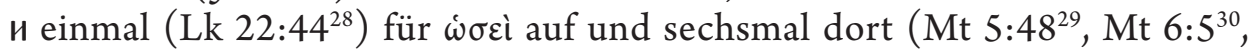

${ }^{23}$ Die Zählungen schließen die zweifelhaften Fälle, die in den Tabellen je angemerkt sind, mit ein.

${ }^{24} \mathrm{CCMH}$ : Assemanianus und Zographensis defekt.

${ }^{25}$ CCMH: Assemanianus ebenso ґako n, Zographensis defekt.

${ }^{26} \mathrm{CCMH}$ : Assemanianus defekt, Zographensis ohne $\boldsymbol{n}$.

27 CCMH: Assemanianus ohne $\mathbf{u}$, Zographensis defekt.

${ }^{28}$ CCMH: Assemanianus ohne $\boldsymbol{u}$, Zographensis mit $\boldsymbol{u}$.

${ }^{29}$ CСMH: Assemanianus ґакожє и, Zographensis defekt.

${ }^{30} \mathrm{CCMH}$ : Assemanius ebenso tako h, Zographensis defekt. 


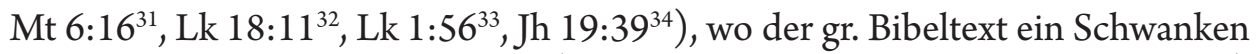

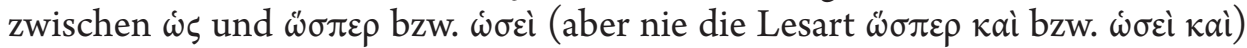
sehen läßt.

Die zwei Entsprechungen von rako u zur Vergleichspartikel $\dot{\omega} \varsigma_{\text {„wie“ }}$ sind beide semantisch als ,wie auch" ${ }^{35}$ klar motiviert, und da es sich um zwei synoptische Stellen handelt, deren dritter synoptischer Partner (Lk 6:10) direkt auf $\omega \varsigma$ кaì zurückgehen dürfte oder zumindest könnte, handelt es sich bei den zwei Entsprechungen $\dot{\omega} \zeta=\mathrm{r} \boldsymbol{\mathrm { l }} \boldsymbol{\mathrm { k }} \mathrm{\textrm {H }}$ nicht nur um denselben pragmatischen Sprechakt, sondern auch um eine möglicherweise sich innerhalb der aksl. Abschreibetradition einstellende Kontamination aller drei Stellen. Mit anderen Worten: Das Auftreten von rako и für gr. $\dot{\omega} \zeta$ gibt keine andere Semantik preis, als es die Bezeugungen von

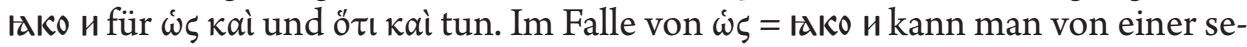
mantischen folgenlosen, höchstens die Zusammengehörigkeit des Verglichenen noch betonenden Variante reden.

Dasselbe läßt sich auch von den 5 Fällen sagen, wo rakoжє и ohne Bezug zu einem gr. kaì im Marianus aufscheint. Alle Stellen stammen aus demselben Kapitel des Jh-Evangeliums ${ }^{36}$ als Entsprechungen für $\kappa a \theta \dot{\omega} \varsigma$, welches typischerweise in Wendungen der Art „wie geschrieben war" oder „wie er gesagt hatte“, also in Verbindungen mit einem Sprechaktverb gebräuchlich ist. Die auf diskursiv vorerwähnte Fakten verweisende Semantik von $\kappa a \theta \dot{\omega} \varsigma$ wird in der perseverierten Übersetzung mit rako $\mathbf{~}$ an dieser speziellen Stelle in Jh durch die Modalpartikel же betont und läßt sich problemlos als verstärkte Vergleichssemantik „wie auch“ erklären ${ }^{37}$.

${ }^{31} \mathrm{CCMH}$ : Assemanianus ohne $\mathbf{n}$, Zographensis defekt.

${ }^{32}$ CCMH: Assemanianus und Zographensis ebenso rako $\mathrm{n}$.

${ }^{33}$ CCMH: Assemanianus und Zographensis beide ohne $\boldsymbol{n}$.

${ }^{34}$ CCMH: Assemanianus ebenso IAKO $\mathrm{n}$, Zographensis defekt.

${ }^{35}$ In Jh 13:15 zeigt sich die Bedeutung von gr. kaì bzw. aksl. и als "und, auch“ deut-

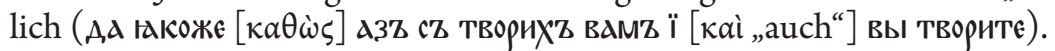

${ }^{36}$ Dass die gr. Vergleichspartikeln sehr ungleich über die Evangelien verteilt sind mit einem deutlichen Übergewicht in Mt, was $\dot{\omega} \zeta, \dot{\omega} \sigma \pi \varepsilon \rho$ und $\dot{\omega} \pi \omega \varsigma$ betrifft, während $\dot{\omega} \sigma \varepsilon \grave{\text { un }}$ und vor allen Dingen $\kappa a \theta \dot{\omega} \zeta$ in den anderen Evangelien (auch gerade bei Jh) angetroffen werden, macht stutzig, doch müssen Untersuchungen zum (semitischen?) Stil von Mt der Bibelwissenschaft überlassen werden.

${ }^{37}$ Auffallend ist, dass der Codex Assemanianus (Zographensis defekt) immerhin drei Mal (+A) mit dem Marianus übereinstimmt: А, БжА,АТZ єд,ино накожє и мы (Jh

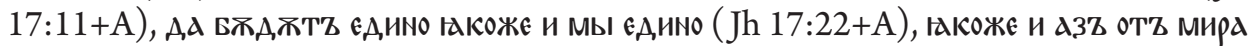

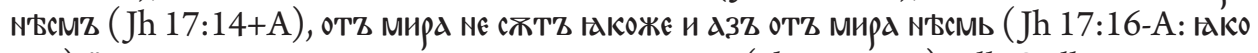

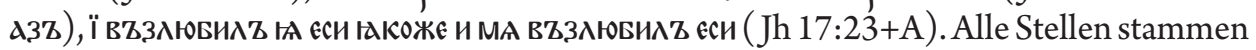
aus derselben Textsorte (Gebet) und zeigen deutliche Formulierungsparallelen; vielleicht ist hier mündliche, im liturgischen Gebrauch geschehene Prägung für die

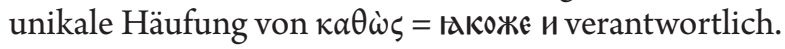




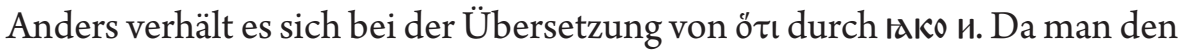
zweiten Beleg ( Jh 6:26) als unsicher ausschließen muss, bleibt nur Lk 16:8 übrig, wo tako $\mathrm{n}$ semantisch nicht leicht erklärt wird. Es handelt sich um das Gleichnis vom untreuen Verwalter (Lk 16:1-9), in welchem ein bestechlicher Finanzverwalter als „klug handelnd“ gelobt wird. Der Vers lautet:

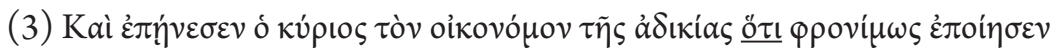

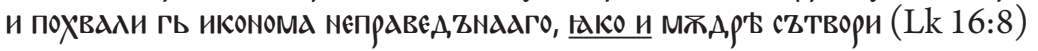

Die versuchsweise Übersetzung des aksl. Textes „dass/weil er auch klug gehandelt habe" impliziert einen rationalisierenden Eingriff des aksl. Übersetzers in den Bibeltext, als ob er mit dem Adverb ,auch“ die Alternative öffne, dass der Verwalter „einerseits“ verbrecherisch, andererseits „auch“ klug im Verfolgen seines Eigennutzes war. Es ist aber angesichts des schwankenden Erscheinens von tako $\mathbf{~}$ in den anderen aksl. Bibelübersetzungen ausgeschlossen, das tako $\mathbf{~}$ an einer bestimmten Stelle mit schwerer exegetischer Bedeutung zu beladen. Trotzdem ist klar, dass im Gegensatz zu den Stellen $\dot{\omega} \varsigma=\ltimes \kappa 0$ и „wie auch“, $\kappa a \theta \grave{\omega} \varsigma=$ ғакожє $\mathbf{n}$,wie auch“ und im Gegensatz zu der (wohl verdorbenen) Stelle ö $\tau \iota=$

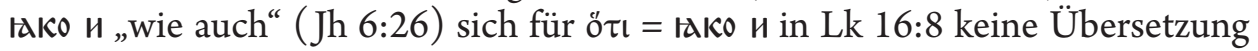
denken läßt, welche nur die Vergleichssemantik unterstützend betont. Wer in Lk 16:8 ein „auch“ einführt, berührt die Logik der Prädikation.

Das in der Bedeutung einer Konjunktion (,und“, ,auch“) bzw. eines Adverbs (,auch“) in Lk 16:8 schwer übersetzbare rako и teilt dieses Schicksal mit den übrigen Fundstellen für taks и im Marianus, die viermal mit $\omega \sigma \pi \varepsilon \rho$ (Mt 5:48 ${ }^{38}$, Mt

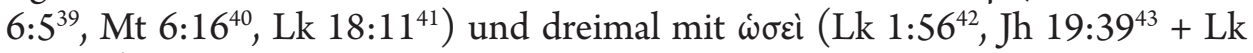
$22: 44^{44}$ ) verbunden sind. Besonders in Verbindung mit $\dot{\omega} \sigma \varepsilon \dot{\imath}$ will и in der Bedeutung „und, auch“ nicht einleuchten, denn $\dot{\omega} \sigma \varepsilon \dot{~ i s t ~ e i n m a l ~ d a s ~ G e g e n s t u ̈ c k ~ z u m ~ d t . ~}$

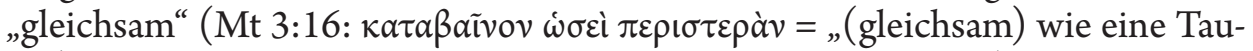
be") und dient spezifisch zur Angabe ungefährer Zahlangaben (,um die sechste Stunde"), und die Beispiele für die Äquivalenz $\dot{\omega} \sigma \varepsilon \grave{l}=$ זako $\mathrm{n}$ machen (fast) keine Ausnahme: „etwa drei Monate“ (Lk 1:56), „etwa hundert Liter“ (Jh 19:39). Es

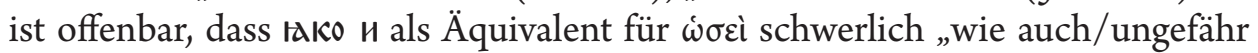
auch" bedeuten kann und sich also die semantische Funktion des и nicht gut erklären läßt. Dieselbe Schwierigkeit bietet auch der Vergleich in Lk 22:44:

38 CСMH: Assemanianus taкожє и, Zographensis defekt.

$39 \mathrm{CCMH}$ : Assemanianus ebenso rako и, Zographensis defekt.

$40 \mathrm{CCMH}$ : Assemanianus ohne и, Zographensis defekt.

${ }^{41} \mathrm{CCMH}$ : Assemanianus ebenso tako и, Zographensis fehlt.

42 CCMH: Assemanianus fehlt, Zographensis ohne и.

43 CCMH: Assemanianus ebenso raко и, Zographensis defekt.

${ }^{44} \mathrm{CCMH}$ : Assemanianus ohne и, Zographensis ebenso ґако и. 


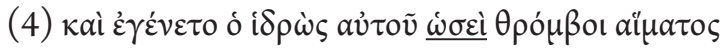

ї БыстZ потZ єго Іако и КАПлА КрZве (Lk 22:44)

Ein Vergleich kann theoretisch auf zwei Arten ausgedrückt werden: der Schweiß kann einen realen Vergleich „wie“ Blutstropfen hervorrufen, oder aber einen hypothetischen „gleichsam wie“ bzw. „als ob" der Schweiß Blutstropfen wäre. Wir werden nachher (Abschnitt 3) noch Zitate bringen, wo m. E. für tako $\mathbf{n}$ ausschließlich eine Übersetzung als hypothetischer Vergleich möglich ist, wie jedenfalls auch bei Satz (4) ein hypothetischer Vergleich naheliegend ist. Deut-

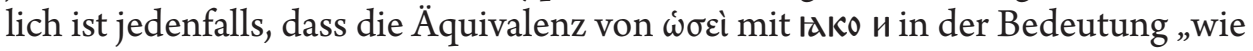
auch" hier ausgeschlossen ist.

In dieselbe Richtung des hypothetischen Vergleiches deuten auch die Äquivalenzen von $\ddot{\omega} \sigma \pi \varepsilon \rho$ mit tako и. Es ist auffällig, dass זako mit dem Zusatz и für $\ddot{\omega} \sigma \pi \varepsilon \rho$ - jedenfalls im Codex Marianus - nur dort eingetreten ist, wo auf $\ddot{\omega} \sigma \varepsilon \varepsilon \rho$ eine Nominalgruppe folgt, während Wendungen mit folgender Partikel $\omega \sigma \pi \varepsilon \rho$

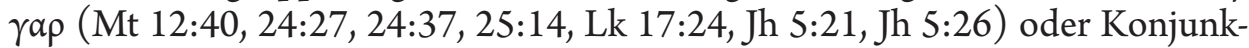

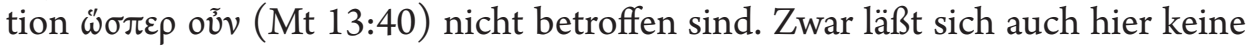
echte Regelmäßigkeit sehen, indem (nach dem gr. Text von SLB zu urteilen) der Marianus drei Male die Verbindung von $\omega \sigma \pi \varepsilon \rho$ + Nominalgruppe auch ohne folgendes и ӥbersetzt (aber siehe dafür den Assemanianus ${ }^{45}$ ), jedoch ist allen Stellen mit $\ddot{\omega} \sigma \tau \varepsilon \rho=$ r $\mathbf{k} \kappa \mathrm{n}$ gemeinsam, dass bei der Übersetzung die Semantik „wie und/auch" (mit Ausnahme von Mt 5:48) unangebracht ist. Vielmehr scheint es um die Determination der Nominalgruppe zu gehen: das Nomen in ғако и отецZ ваш乙 нвскы (Mt 5:48) = „seid vollkommen, wie (auch) euer himmlischer Vater“ ist wegen des Possessivpronomens und qua Unikalität per se definit, aber auch in זако и лицем'Бри (Mt 6:5) = ґко и ипокрити [Mt 6:16] = „wie diese Heuchler" und ґако и прочии чхвци (Lk 18:11) = „wie diese anderen Menschen da“ werden identifizierbare, wenngleich indefinit gelassene Entitäten aus dem Wahrnehmungsfeld der erzählten Person referenziert, wobei sich eine Übersetzung mit „auch“ verbietet (der Sprecher grenzt sich im Gegensatz zu einer Übereinstimmung vielmehr von den anderen Menschen $a b$ ). Es ließe sich leicht für die schon besprochenen Beispiele von $\dot{\omega} \varsigma$ = ז $\mathrm{\kappa} \kappa \mathrm{n}$ dasselbe Phänomen der Determiniertheit behaupten, da die in diesen Beispielen bezeichnete Entität („die andere Hand“) ebenfalls qua Konzeptwissen definit ist, aber während man bei $\dot{\omega} \zeta=$ rako и einen möglichen Bezug zur Determination nicht herstellen muss, drängt er sich bei $\omega \sigma \pi \varepsilon \rho=$ rako $\mathrm{n}$ fast auf.

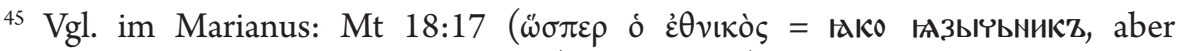

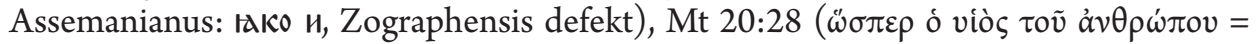

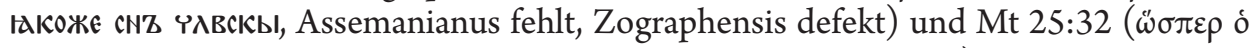

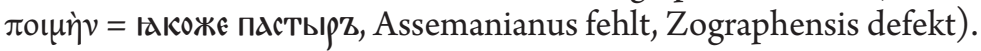




\section{3. tako $и$ in anderen kirchenslavischen Texten}

Die Verwendung von זیке и im Codex Marianus zeigt, dass sich die Übersetzungsäquivalente von $\dot{\omega} \sigma \varepsilon \dot{/} / \ddot{\omega} \sigma \pi \varepsilon \rho=$ זako и nicht der Semantik ,wie auch“ einfügen bzw. dass и „,und, auch“ bei der Übersetzung unberücksichtigt bleiben muss. Die nachfolgende Tabelle führt nun einige zufällig gewählte Zitate aus der kirchenslavischen Literatur an, in welchen tako $\mathrm{n}$ ebenfalls nicht mit „wie auch/ wie und“, sondern ausschließlich mit „gleichsam als“ übersetzt werden kann, in denen also ein positiver Vergleich unmöglich ist und nur ein hypothetischer Vergleich „als ob“ statthaben kann:

(5) Нь самь ғако и слнще тоу озариль юси (Zhivova 2016, 74)

(6) Тако ға оуловиста ғако и рыбы, словесньною мръжењқ (Stančev, Calusio 2017, 84)

(7) Тому бо подъ крилома ғако и голубь въскормленъ бы[с] дхивною пищею (Stančev, Calusio 2017, 115)

(8) мноsи бо ғако и $\bar{w}$ самого чрьтога црева прихождахоy (Pavlikianov 2014, $595)$

(9) и пожеже всеиі и быс[т] ғако и прахь (Christova-Šomova 2013, 372)

(10) и придъ шгнь с нбсь. и пожеже всеиі и быс[т] нако и прахь (TăpkovaZaimova, Miltenova 2011,439)

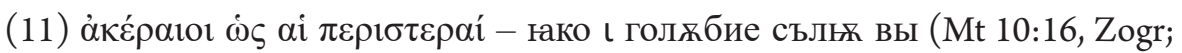
Penkova 2016, 288)

Es ist offenkundig, dass in Beispielsätzen (5)-(11) eine Übersetzung „wie auch" absurd wirkt:

(ad 5) ? ,aber selbst hast du dort wie auch die Sonne geglänzt ${ }^{*}$ - ist von zwei Sonnen die Rede, oder sollte es nicht besser heißen, hast gleichsam wie die Sonne geglänzt'? (ad 6) ? ,So habt ihr beide sie gefangen wie auch Fische... - wurden Menschen und auch Fische gefangen oder wurden nicht eher, Menschen gleichsam wie Fische gefangen'?

(ad 7$)$ ? ,Denn unter diesem Flügelpaar wurdest du wie auch eine Taube mit geistiger Nahrung genährt' - wahrscheinlich wurde nicht auch noch eine Taube genährt, sondern der Angesprochene wurde "gleichsam wie eine Taube" genährt.

(ad 8) ? ,viele nämlich kamen wie auch vom kaiserlichen Vorhof' - woher kamen sie noch? Der Satz ist metaphorisch zu verstehen "gleichsam vom kaiserlichen Vorhof". (ad 9) ? , und verbrannte alles und es wurde wie auch Asche - und es wurde „gleichsam zu Asche" (ad 10) idem. 
(ad 11) ? ,wie auch die Tauben schicke ich euch ${ }^{\text {- }}$ - der Codex Zographensis korrumpiert den Text von Mt 10:16 (,schicke euch ... unschuldig wie die Tauben'), indem er das Prädikat „unschuldig“ eliminiert, und da nicht bekannt ist, wie man typischerweise ,Tauben schickt' rettet er sich in da verbliebene Prädikat „gleichsam wie Tauben“.

Natürlich gibt es Zweifelsfälle, die zwischen dem Verständnis eines positiven und eines hypothetischen Vergleiches schwanken. Der Leser wird möglicherweise schon bei Sätzen (1) bis (11) das eine oder andere Beispiel anders auffassen, jedoch - dies wäre die Widerlegung vorliegender Thesen - die zwingende Annahme hypothetischer Vergleiche in Sätzen (1) bis (11) nicht grundsätzlich bezweifeln. Ein Beispielsatz wie (12) stellt nochmals die ganze Schwierigkeit vor Augen. Man kann ebenso gut sagen, jemand sei „wie tot" als jemand sei „gleichsam tot", denn dieser Vergleich kann immer nur eine metaphorische, aber nie reale Prädikation vollziehen: mit dem Tot kann man keine anderen objektiven Übereinstimmungen haben, als tatsächlich tot $\mathrm{zu}$ sein (man kann nicht ein wenig tot sein). Folglich sind „wie tot" oder "gleichsam tot" semantisch identische Prädikationen; aber die Übersetzung ,wie auch tot" (Zographensis) ist absurd:

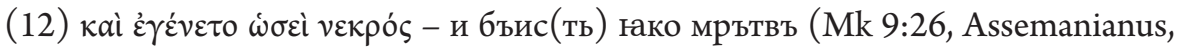
Marianus, Savvina kniga) rako I (Zographensis)

Cejtlin, Večerka, Bláhová (1994, 793), denen ich Beispiel (12) samt den Lesarten entnehme, führen es bereits als Beispiel für einen hypothetischen („как буато, jakoby“) Vergleich an, aber mir scheint, dass ausgerechnet Satz (12) als Beispiel für einen hypothetischen Vergleich weniger beweiskräftig ist, da ein realer Vergleich hier unmöglich ist: der Tot kann immer nur hypothetisch verglichen werden - sonst ist er eingetreten. Wenn man sich aber fragt, warum die Lesarten zwischen rako und rako $\mathrm{H}$ in (12) schwanken, denke man an (5) bis (11), wo ein realer Vergleich „wie auch“ seltsam wirkt. Dennoch muss man zugeben, dass eine geregelte semantische Verteilung zwischen rako und rako $\mathbf{~}$ schwer zu sehen ist und immer auch gr. Vorlagen hier hineinspielen können, die nur (noch) nicht bekannt sind, wie etwa im folgenden Zitat aus den Dialogen Gregors, dessen zweifache Übersetzung sich offenbar nicht nur bei rako $и$ im Bezug auf die gr. Vorlage unterscheidet:

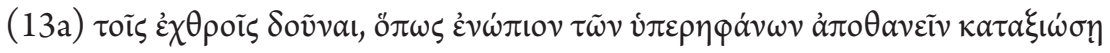

(13b) враго[м] дати м'Всто, гако пре[А] [горды] ими Вммр'Тти спод,овит са (gr., aksl. Diddi 2001, 134)

(13c) врагом Адтти м'ссто, накW и оумрети пре[А] ними спод,овитиса (VMС̌ 11. März, Uspenskij spisok 126c:10-12, ed. Weiher et al., 1997) 
Das textologisch schwierige Beispiel (13) erinnert daran, dass nicht jedes Auftreten von raks $\mathrm{n}$ als Anzeige eines hypothetischen Vergleiches genommen werden darf. Es ist vielmehr auch immer die Semantik der Stelle zu betrachten, und zwar unter der Vorgabe, dass „wie“ einen Vergleich einleitet, „gleichsam wie“ aber eine Metapher. Von einem realen Vergleich „er sah sein Gesicht wie im Spiegel“ kann man immer zu einem hypothetischen Vergleich wechseln „er sah sein Gesicht gleichsam wie im Spiegel“" (denn der Weg zur Metapher steht immer offen) und daher sind diese Proben der Übersetzbarkeit nicht aussagekräftig. Die harte Probe, ob man es bei זaкo n mit einem hypothetischen Vergleich zu tun habe, liegt nicht in der Ersetzbarkeit von „wie“ durch „gleichsam als“, sondern vielmehr in der Übersetzbarkeit der Konjunktion: Wenn nicht „wie auch/ und" übersetzt werden kann, darf man sich fragen, welche Mechanismen - denn semantische Intentionen können es nicht sein - die Schreiber bewogen haben mögen, den Vergleich mit einer semantisch uninterpretierbaren Konjunktion zu markieren.

\section{Hypothese}

Im schmalen Raum eines Aufsatzes kann nicht beantwortet werden, was objektiv darzulegen nur auf breiter, historisch, dialektal und orthographisch spezifizierter Materialbasis möglich wäre: $\mathrm{Ob}$ die wörtlich unmöglich zu übersetzenden Verbindungen von rako $\mathrm{n}=$,wie und/auch" nicht mit der Zeit immer mehr die Bedeutung des hypothetischen Vergleiches annehmen und im späteren Kirchenslavischen bevorzugt in dieser Funktion verwendet werden? Impressionistische Beispiele wie in den Sätzen (5) bis (11) sind wohl geeignet, die Frage zu legitimieren angesichts der aus dem Codex Marianus erhobenen Materialanalyse, welche zeigt, dass die Form łako n einerseits wörtlich die gr. Verbindung „wie auch“ übersetzt und so frequent im Schrifttum vorliegt, dass aber andererseits die Verbindung der Konjunktion $\boldsymbol{и}$ mit rako im Umkreis der

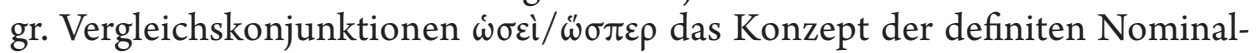
referenz berührt, was in der syntaktischen Form des Vergleiches also bedeutet, dass die Frage, ob eine konkrete Übereinstimmung des Vergleichsprädikates mit seinem Nomen ("x ist wie $\mathrm{y}^{\prime)}$ ) oder aber nur eine unbestimmt-metaphorische („x ist gleichsam wie $\left.\mathrm{y}^{\prime \prime}\right)$ angezielt wird. Die Verbindung rako $\mathrm{n}$ berührt

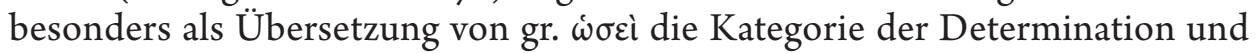
markiert sich damit selbst, ohne dass damit gesagt sein soll, dass rako и schon im Aksl. geregelt einen hypothetischen Vergleich ausdrücke. Es erscheint aber möglich, dass die Konjunktion и zunächst einfach analogisch wie nach ö $\tau \iota$ auch an rako als Übersetzung von $\tilde{\omega} \sigma \pi \varepsilon \rho / \dot{\omega} \sigma \varepsilon \dot{~ g e h a ̈ n g t ~ w u r d e, ~ d o r t ~ a b e r ~ v . a . ~ a l s ~ A ̈ q u i-~}$ valent für $\dot{\omega} \sigma \varepsilon \grave{~ d a s ~ P r o b l e m ~ d e r ~ s e m a n t i s c h e n ~ U n v e r t r a ̈ g l i c h k e i t ~ a u f w a r f, ~ w e l-~}$ ches dann vielmehr selbst zum Kennzeichen der Wendung rako $\mathrm{n}$ wurde, indem 
diese sich mit der Zeit als Ausdruck für hypothetische Vergleiche einbürgerte. Es läge also ein Fall von Grammatikalisierung vor, der sich im Schrifttum vollzieht, indem die Abschreiber einer zunächst absichtslos auftretenden Variante mit der Zeit einen neuen Sinn geben.

\section{Literatur}

Bauer, W. (1988). Griechisch-deutsches Wörterbuch zu den Schriften des Neuen Testaments und der frühchristlichen Literatur. 6. Aufl., von K. Aland, B. Aland (ed.). Berlin, Boston: De Gruyter.

CCMH = Lindstedt, J. (ed.) (1986-2010). Corpus Cyrillo-Methodianum Helsingiense. An Electronic Corpus of Old Church Slavonic Texts. <http://www.helsinki.fi/slaavilai$\mathrm{set} / \mathrm{ccmh} />$ (zuletzt gesehen am 14.11.2020).

Cejtlin, R.M., Večerka, R., Bláhová, E. (ed.) (1994). Staroslavjanskij slovar' (po rukopisjam X-XI vekov). Moskva: Russkij jazyk.

Christova-Šomova, I. (2013). Staroslavjanskaja sinonimija v rannich perevodach služb $v$ prazdničnych i služebnych mi nejach. Christianskij Vostok, Novaja Serija, 6, 391-400.

Daiber, T. (2009). Direkte Rede im Russisch-Kirchenslavischen. Zum pragmatischen Wert des jako recitativum. In: Text - Sprache - Grammatik. Festschrift für Eckhard Weiher. J. Besters-Dilger, A.Rabus (ed.). München: Sagner, 363-386. (= Die Welt der Slaven, Sammelbände 39).

Daiber, T. (2013). Modalität der Redewiedergabe (Jako recitativum im Codex Marianus). In: Deutsche Beiträge zum 15. Internationalen Slavistenkongress. S. Kempgen, M. Wingender, N. Franz, M. Jakiša (ed.). Minsk. München: Sagner, 109-116. (= Die Welt der Slaven, Sammelbände 50).

Diddi, C. (ed.) (2001). Paterik Rimskij. Dialogi Grigorija Velikogo v drevneslavjanskom perevode. Moskva: Indrik. (= Pamjatniki drevnej pis'mennosti. Issledovanija, teksty).

Jagić, V. (ed.) (1960). Quattuor Evangeliorum versionis palaeoslovenicae Codex Marianus Glagoliticus characteribus cyrillicis transcriptum [zuerst 1883; Nachdruck]. Graz: Akademische Druck- und Verlagsanstalt.

Nestle, Alland (2012) = Aland, B., Aland, K., Karavidopoulos, J., Martini, C.M., Metzger, B.M. (ed.), Novum Testamentum Graece. Based on the work of E. and E. Nestle. 28. Aufl. Stuttgart: Deutsche Bibelgesellschaft.

Pavlikianov, C. (2014). The Mediaeval Greek and Bulgarian Documents of the Athonite Monastery of Zographoe (980-1600). Critical Edition and Commentary of the Texts. Sofija: Kliment Ohridski Univ. (= Universitetska biblioteka 512).

Penkova, P. (2016). Sveti Atanasij Aleksandrijskij (Veliki). Treto slovo protiv Arianite. Izsledvane $i$ izdanie na teksta. Sofija: Valentin Trajanov.

Robinson, M.A., Pierpont, W.G. (2005). The New Testament in the Original Greek. Byzantine Textform. Southborrow (MA): Chilton Books.

Rondestvedt, K.A. (1986). Definite, indefinite and related pragmatic categories in early original Slavic. Diss. phil., Univ. Chicago.

SBL = Holmes, M.W. (ed.) (2010). The Greek New Testament. Atlanta: Society of Biblical Literature and Logos Bible Software. 
Stančev, K., Calusio, M. (ed.) (2017). San Clemente di Ocrida: Allievo e Maestro. Nell'undicesimo centenario del Beato Transito (916-2016). Milano: Biblioteca Ambrosiana. (= Slavica Ambrosiana 7).

Tăpkova-Zaimova, V., Miltenova, A. (2011). Historical-apocalyptic Literature in Byzantium and Medieval Bulgaria. Sofia: East-West Publishers.

Tommola, H. (2014). Nedostovernoe sravnenie v russkom i drugich slavjanskich jazykach po dannym parallel'nogo korpusa. In: Grammaticalization and Lexicalization in the Slavic Languages. M. Nomachi, A. Danylenko, P. Piper (ed.). München, Berlin, Washington: Kubon \& Sagner, 301-315. (= Die Welt der Slaven, Sammelbände 55).

Weiher, E., Šmidt, S.O., Škurko, A.I., Daiber, T., Daiber, Y., Dianova, T.V., Keller, F., Kobjak, N.A., Kostjuchina, L.M., Minčeva, A., Pliguzov, A.I., Serebrjakova, E.I., Voss, C., Šul'gina, E.V. (ed.) (1997). Die Großen Lesemenäen des Mitropoliten Makarij. 1.-11. März. Freiburg i. Br.: Weiher. (= Monumenta Linguae Slavicae Dialecti Veteris, Fontes et Dissertationes 39).

Zhivova, M.V. (2016). La ricezione del culto dei pontefici romani nella tradizione liturgicoletteraria medievale della Slavia Orthodoxa. Diss. phil., Univ. Pisa. 